Int. J. Contemp. Math. Sci., Vol. 2, 2007, no. 10, 469 - 474

\title{
Neighborhoods of a Certain Class of Analytic Functions with Negative Coefficients
}

\author{
H. Özlem Güney and S. Sümer Eker \\ University of Dicle, Faculty of Science and Arts \\ Department of Mathematics, 21280, Diyarbakır, Turkey \\ ozlemg@dicle.edu.tr \& sevtaps@dicle.edu.tr
}

\begin{abstract}
By making use of the familiar concept of neighborhoods of analytic functions, we prove several inclusion relations associated with the $(n, \delta)$ neighborhoods of various subclass of univalent functions with negative coefficients.
\end{abstract}

\section{Mathematics Subject Classification: 30C45}

Keywords: analytic function, negative coefficient, $(n, \delta)$-neighborhood

\section{Introduction}

Let $\mathcal{A}(n)$ denote the class of functions $f(z)$ of the form

$$
f(z)=z-\sum_{k=n+1}^{\infty} a_{k} z^{k} \quad\left(a_{k} \geq 0, n \in \mathbb{N}:=\{1,2,3, \ldots\}\right)
$$

which are analytic in the open unit disk $\mathbb{U}=\{z \in \mathbb{C}:|z|<1\}$.

For any $f(z) \in \mathcal{A}(n)$ and $\delta \geq 0$ we define

$$
\mathcal{N}_{n, \delta}(f)=\left\{g \in \mathcal{A}(n): g(z)=z-\sum_{k=n+1}^{\infty} b_{k} z^{k}, \sum_{k=n+1}^{\infty} k\left|a_{k}-b_{k}\right| \leq \delta\right\}
$$

which was called $(n, \delta)$-neighborhoods of $f(z)$. So, for $e(z)=z$, we see that

$$
\mathcal{N}_{n, \delta}(e)=\left\{g \in \mathcal{A}(n): g(z)=z-\sum_{k=n+1}^{\infty} b_{k} z^{k}, \sum_{k=n+1}^{\infty} k\left|b_{k}\right| \leq \delta\right\}
$$


The concept of neighborhoods was firstly by A.W.Goodman[1] and then generalized by ST.Ruscheweyh [2].

The main object of the present paper is to investigate the neighborhoods of the following subclass of class $\mathcal{A}(n)$ of univalent functions with negative coefficients.

A function $f(z) \in \mathcal{A}(n)$ is said to be in the class $\mathcal{P}_{\gamma}(n, \lambda, \alpha, r)$ if it satisfies

$$
\operatorname{Re}\left\{z \frac{2 \gamma \lambda r f^{\prime \prime}(z) z^{2 r-1}+\lambda \gamma f^{\prime \prime \prime}(z) z^{2 r}+(\lambda-\gamma) r f^{\prime}(z) z^{r-1}+(\lambda-\gamma) f^{\prime \prime}(z) z^{r}+(1-\lambda+\gamma) f^{\prime}(z)}{\gamma \lambda z^{2 r} f^{\prime \prime}(z)+(\lambda-\gamma) f^{\prime}(z) z^{r}+(1-\lambda+\gamma) f(z)}\right\}>\alpha
$$

for some $r=1,2, \ldots, \alpha(0 \leq \alpha<1), \lambda, \gamma(0 \leq \gamma \leq \lambda \leq 1)$ and for all $z \in$ $U$ [7]. We note that $\mathcal{P}_{0}(n, \lambda, \alpha, r) \equiv \mathcal{P}(n, \lambda, \alpha r)$. The classes $\mathcal{P}(n, \lambda, \alpha r)$ and $\mathcal{P}(n, \lambda, \alpha r, r)$ were studied by O.Altintas [3] and M.Kamali et al. [4], respectively. $\mathcal{P}_{\gamma}(n, \lambda, \alpha, r)$ was introduced by M.Kamali et al.[5]. The class $\mathcal{P}_{\gamma}(n, \lambda, \alpha, r)$ is generalization of $\mathcal{P}(n, \lambda, \alpha r)$ by O.Altintas [3].

\section{A Set of Inclusion Relations Involving $\mathcal{N}_{n, \delta}(e)$}

In our investigation of the inclusion relations involving $\mathcal{N}_{n, \delta}(e)$, we shall require the following Lemma which was proved in [7].

Lemma Let $f(z) \in \mathcal{A}(n)$ is in the class $\mathcal{P}_{\gamma}(n, \lambda, \alpha, r)$ if and only if

$$
\begin{gathered}
\sum_{k=n+1}^{\infty}\{(k-\alpha)[(k-1)(\lambda \gamma k+\lambda-\gamma)+1] k(r-1)[2 \lambda \gamma(k-1)+\lambda-\gamma]\} a_{k} \\
\leqq(1-\alpha)+(\lambda-\gamma)(r-1)
\end{gathered}
$$

Our first inclusion relation involving $\mathcal{N}_{n, \delta}(e)$ is given by the following:

Theorem 1 Let

$$
\delta_{1}=\frac{(n+1)(1-\alpha)}{(n+1-\alpha)[n(\lambda \gamma(n+1)+\lambda-\gamma)+1]}
$$

and

$$
\begin{gathered}
\delta_{2}=\frac{\{(n+1)[n(\lambda \gamma(n+1)+\lambda-\gamma)+1]+(n+1)(r-1)[2 \lambda \gamma n+\lambda-\gamma]\}}{\{(n+1-\alpha)[n(\lambda \gamma(n+1)+\lambda-\gamma)+1]+(n+1)(r-1)[2 \lambda \gamma n+\lambda-\gamma]\}} \times \\
\frac{[(1-\alpha)+(\lambda-\gamma)(r-1)]}{\{(r-1)[2 \lambda \gamma n+\lambda-\gamma]+[n(\lambda \gamma(n+1)+\lambda-\gamma)+1]\}} .
\end{gathered}
$$

then

$$
\mathcal{P}_{\gamma}(n, \lambda, \alpha, 1) \subset \mathcal{N}_{n, \delta_{1}}(e)
$$


and

$$
\mathcal{P}_{\gamma}(n, \lambda, \alpha, r) \subset \mathcal{N}_{n, \delta_{2}}(e) \text { for } r=2,3, \ldots,
$$

respectively.

Proof For $f \in \mathcal{P}_{\gamma}(n, \lambda, \alpha, 1)$, Lemma is,

$$
\sum_{k=n+1}^{\infty}(k-\alpha)[(k-1)(\lambda \gamma k+\lambda-\gamma)+1] a_{k} \leqq(1-\alpha)
$$

Therefore, Lemma immediately yields,

$$
(n+1-\alpha)[n(\lambda \gamma(n+1)+\lambda-\gamma)+1] \sum_{k=n+1}^{\infty} a_{k} \leqq(1-\alpha) .
$$

so that

$$
\sum_{k=n+1}^{\infty} a_{k} \leqq \frac{1-\alpha}{(n+1-\alpha)[n(\lambda \gamma(n+1)+\lambda-\gamma)+1]}
$$

On the other hand, we also find (2.2) and (2.3) that

$$
\begin{gathered}
\sum_{k=n+1}^{\infty} k[(k-1)(\lambda \gamma k+\lambda-\gamma)+1] a_{k}+\sum_{k=n+1}^{\infty}(-\alpha)[(k-1)(\lambda \gamma k+\lambda-\gamma)+1] a_{k} \\
\leqq(1-\alpha)[n(\lambda \gamma(n+1)+\lambda-\gamma)+1] \sum_{k=n+1}^{\infty} k a_{k} \\
\leqq(1-\alpha)+\alpha[n(\lambda \gamma(n+1)+\lambda-\gamma)+1] \sum_{k=n+1}^{\infty} a_{k} \\
\sum_{k=n+1}^{\infty} k a_{k} \leqq \frac{(n+1)(1-\alpha)}{(n+1-\alpha)[n(\lambda \gamma(n+1)+\lambda-\gamma)+1]}=\delta_{1}
\end{gathered}
$$

and for $f \in \mathcal{P}_{\gamma}(n, \lambda, \alpha, r)$ Lemma immediately yields

$$
\{(n+1-\alpha)[n(\lambda \gamma(n+1)+\lambda-\gamma)+1]+(n+1)(r-1)[2 \lambda \gamma n+\lambda-\gamma]\} \sum_{k=n+1}^{\infty} a_{k}
$$




$$
\leqq(1-\alpha)+(\lambda-\gamma)(r-1)
$$

so that

$$
\begin{gathered}
\sum_{k=n+1}^{\infty} a_{k} \leqq \\
\frac{(1-\alpha)+(\lambda-\gamma)(r-1)}{\{(n+1-\alpha)[n(\lambda \gamma(n+1)+\lambda-\gamma)+1]+(n+1)(r-1)[2 \lambda \gamma n+\lambda-\gamma]\}} .
\end{gathered}
$$

On the other hand, we also find (2.1) and (2.4) that

$$
\begin{gathered}
(r-1)[2 \lambda \gamma n+\lambda-\gamma]+[n(\lambda \gamma(n+1)+\lambda-\gamma)+1] \sum_{k=n+1}^{\infty} k a_{k} \\
\leqq(1-\alpha)+(\lambda-\gamma)(r-1)+\alpha[n(\lambda \gamma(n+1)+\lambda-\gamma)+1] \sum_{k=n+1}^{\infty} a_{k} \\
\leqq(1-\alpha)+(\lambda-\gamma)(r-1) \\
+\frac{\alpha[n(\lambda \gamma(n+1)+\lambda-\gamma)+1][(1-\alpha)+(\lambda-\gamma)(r-1)]}{\{(n+1-\alpha)[n(\lambda \gamma(n+1)+\lambda-\gamma)+1]+(n+1)(r-1)[2 \lambda \gamma n+\lambda-\gamma]\}}
\end{gathered}
$$

that is

$$
\begin{gathered}
\sum_{k=n+1}^{\infty} k a_{k} \leq \frac{\{(n+1)[n(\lambda \gamma(n+1)+\lambda-\gamma)+1]+(n+1)(r-1)[2 \lambda \gamma n+\lambda-\gamma]\}}{\{(n+1-\alpha)[n(\lambda \gamma(n+1)+\lambda-\gamma)+1]+(n+1)(r-1)[2 \lambda \gamma n+\lambda-\gamma]\}} \times \\
\\
\frac{[(1-\alpha)+(\lambda-\gamma)(r-1)]}{\{(r-1)[2 \lambda \gamma n+\lambda-\gamma]+[n(\lambda \gamma(n+1)+\lambda-\gamma)+1]\}} .
\end{gathered}
$$

3. Neighborhoods for the class $\mathcal{P}_{\gamma}^{(\beta)}(n, \lambda, \alpha, r)$

In this section, we determine the neighborhoods for the class $\mathcal{P}_{\gamma}^{(\beta)}(n, \lambda, \alpha, r)$ which we define as follows. A function $f(z) \in \mathcal{A}(n)$ is said to be in the class $\mathcal{P}_{\gamma}^{(\beta)}(n, \lambda, \alpha, r)$ if there exists a function $g \in \mathcal{P}_{\gamma}(n, \lambda, \alpha, r)$ such that

$$
\left|\frac{f(z)}{g(z)}-1\right|<1-\beta
$$


for $\beta(0 \leq \beta<1)$ and $z \epsilon U$.

Theorem 2 If $g \in \mathcal{P}_{\gamma}(n, \lambda, \alpha, r)$ and

$$
\beta=1-\frac{\{n(n+2 r-1-\alpha)[\lambda \gamma(n+1)+(\lambda-\gamma)]+(\lambda-\gamma)(r-1)(1-\alpha)\} \delta}{n(n+1)\{\lambda \gamma(n+1)(n+2 r-1-\alpha)+(n+r-\alpha)(\lambda-\gamma)+1\}}
$$

then

$$
\mathcal{N}_{n, \delta}(g) \subset \mathcal{P}_{\gamma}^{(\beta)}(n, \lambda, \alpha, r)
$$

Proof Suppose that $f \in \mathcal{N}_{n, \delta}(g)$. Then we find from (1.2) that

$$
\sum_{k=n+1}^{\infty} k\left|a_{k}-b_{k}\right| \leq \delta
$$

which readily implies the coefficients inequality

$$
\sum_{k=n+1}^{\infty}\left|a_{k}-b_{k}\right| \leq \frac{\delta}{n+1}, n \in \mathbb{N} .
$$

Next, since $g \in \mathcal{P}_{\gamma}(n, \lambda, \alpha, r)$ we have from (2.2)

$$
\begin{gathered}
\sum_{k=n+1}^{\infty} b_{k} \\
\leqq \frac{(1-\alpha)+(\lambda-\gamma)(r-1)}{\{(n+1-\alpha)[n(\lambda \gamma(n+1)+\lambda-\gamma)+1]+(n+1)(r-1)[2 \lambda \gamma n+\lambda-\gamma]\}}
\end{gathered}
$$

so that

$$
\begin{gathered}
\left|\frac{f(z)}{g(z)}-1\right|<\frac{\sum_{k=n+1}^{\infty}\left|a_{k}-b_{k}\right|}{1-\sum_{k=n+1}^{\infty} b_{k}} \\
\leq \frac{\delta}{n+1} \frac{n(n+2 r-1-\alpha)[\lambda \gamma(n+1)+(\lambda-\gamma)]+(\lambda-\gamma)(r-1)(1-\alpha)}{n\{\lambda \gamma(n+1)(n+2 r-1-\alpha)+(n+r-\alpha)(\lambda-\gamma)+1\}}=1-\beta
\end{gathered}
$$

provided that $\beta$ is given precisely by (3.2). Thus, by definition of $\mathcal{P}_{\gamma}^{(\beta)}(n, \lambda, \alpha, r)$, $f \in \mathcal{P}_{\gamma}^{(\beta)}(n, \lambda, \alpha, r)$ for $\beta$ given by (3.2), which evidently completes our proof of Theorem 2. 


\section{References}

[1] A.W.GOODMAN; Univalent Functions and nonanalytic curves, Proc. Amer. Math. Soc.,8(1957),598-601.

[2] ST.RUSHEWEYH;Neighborhoods of univalent functions, Proc. Amer. Math. Soc.,81(1981),521-527.

[3] O.ALTINTAS; On a subclass of certain starlike functions with negative coefficients, Math. Japonica,36(3)(1991),489-495.

[4] M.KAMALI and E.KADIOGLU; A new class of analytic functions with negative coefficients, Math. Balkanica Fasc.,13(1-2)(1999),29-39.

[5] M.KAMALI and E.KADIOGLU;On a subclass of certain starlike functions with negative coefficients, Atti Sem.Math.Fis.Univ.Modena XLVIII (2000), 31-44.

[6] H.SILVERMAN; Univalent Functions with negative coefficients, Proc. Amer. Math. Soc.,51(1975),109-116.

[7] H.ORHAN; A new class of analytic functions with negative coeffcients, Appl.Math. and Comp.,138(2003),531-543.

Received: June 29, 2006 